\title{
Imitation of Honeybee Aggregation with Collective Behavior of Swarm Robots
}

\author{
Farshad Arvin* \\ Department of Computer Engineering, Science and Research Branch, \\ Islamic Azad University, Tehran, Iran \\ E-mail:farshadarvin@yahoo.com \\ Khairulmizam Samsudin, Abdul Rahman Ramli \\ Department of Computer Systems Engineering, University Putra Malaysia, \\ 43400 UPM, Serdang, Selangor,Malaysia \\ E-mail:kmbs@eng.upm.edu.my,arr@eng.upm.edu.my \\ Masoud Bekravi \\ Department of Computer Engineering, Ardabil Branch, \\ Islamic Azad University, Ardabil, Iran \\ E-mail: masoud_bekravi@iauardabil.ac.ir \\ Received 25 September 2010 \\ Accepted 1 June 2011
}

\begin{abstract}
This paper analyzes the collective behaviors of swarm robots that play role in the aggregation scenario. Honeybee aggregation is an inspired behavior of young honeybees which tend to aggregate around an optimal zone. This aggregation is implemented based on variation of parameters values. In the second phase, two modifications on original honeybee aggregation namely dynamic velocity and comparative waiting time are proposed. Results of the performed experiments showed the significant differences in collective behavior of the swarm system for different algorithms.
\end{abstract}

Keywords: Swarm robotics, Honeybee aggregation, Collective behavior, Swarm Intelligence

\section{Introduction}

Cooperation of multiple robots is an interesting area in the robotics field. Performing a joint task with interactions among a large number of simple robots could solve a complex problem in real world application. Furthermore, coordination of the multiple robots for solving a complex problem can be completed in a short period of time. Swarm robotics is a new concept of multi-robot collaboration which is inspired from nature. ${ }^{1}$ It has several differences with ordinary multi-robot researches. ${ }^{2}$ In this regard, Dorigo and Şahin $^{3}$ defined a set of criteria for swarm robotics research as follows:

- Swarm robotics is the study on the large number of simple robots which cooperate to solve a problem.

- Swarm research concentrates on collective behavior of homogeneous robots. Hence, homogeneity and

${ }^{*}$ Farshad Arvin is with the Department of Computer Engineering, Science and Research Branch, Islamic Azad University, Tehran, Iran (E-mail: farshadarvin@yahoo.com) 
scalability are important requirements of swarm robotics.

- Generally, defined task for swarm robotics are hard to solve by a single robot. Therefore, swarm task should be defined based on: (i) a complex task which could not be solved by an individual robot or (ii) if the problem is solvable by an individual, the use of swarm would improve the efficiency to solve the problem.

- Swarm robots have a limited sensing ability and rely on local communication.

Generally, swarm algorithms take inspiration from behaviors of social insects and animals. ${ }^{2}$ Schools of fish, flocks of birds, colonies of termites and ants, ${ }^{4}$ foraging, ${ }^{5}$ and aggregation of honeybees ${ }^{6}$ are examples of swarm behavior. Ant colony optimization and particle swarm optimization are the most successful applications of swarm in problem solving that have been used in variety of applications. ${ }^{7,8,9}$ Aggregation is a natural behavior of social insects and animals to find food or path. ${ }^{10}$ Usually, environmental cues are used as a marker for an optimal zone such as humidity for sow bugs or light and temperature for flies. Conversely, self-organized aggregation is another type of aggregation that does not require any cues, ${ }^{11}$ such as school of fish or aggregation of cockroaches. ${ }^{12}$ In early robotic research by Kube and Zhang, ${ }^{13}$ the light source was employed as the aggregation cue. Melhuish et al. ${ }^{14}$ performed aggregation of robots around an infra-red (IR) transmitter. Each robot after reaching the marked zone with IR starts to play sound similar to frogs and birds. Size of group is estimated based on intensity of choruses. Therefore, based on the estimated swarm size, robots could control the cluster size. Schmickl et al. ${ }^{15}$ presented a cue based aggregation of simple robots that used limit communication. Garnier et al. ${ }^{16}$ simulated cockroaches' aggregation behavior with micro robots. Group of these robots were deployed into circular white arena for 60 minutes. The robots executed proposed aggregation algorithm by Jeanson et al., ${ }^{17}$ which was inspired from cockroach behavior. Based on this algorithm, cockroaches prefer to stay in a peripheral environment with constant time between be in stationary (resting) and in motion. The resting time increases when there are other cockroaches. The aggregation behavior of honeybees is another inspired example of insects' aggregation which has been implemented by swarm micro robots. ${ }^{6}$ According to this aggregation behavior, young honeybees tend to aggregate around a comfortable zone which is an optimal temperature between 34 and $38^{\circ} \mathrm{C} .{ }^{18}$ Details of this algorithm and its implementation will be explained in the following section.

In this paper, an imitation of honeybee aggregation with miniature size mobile robots is presented. The effects of several parameters such as waiting time, arena size, environmental perception, population sizes, and velocity on performance of the swarm system are analyzed. Afterwards, two modifications on original BEECLUST algorithm which are dynamic velocity (DV) and comparative waiting time (CWT) are proposed. These modifications are not in contrast with the swarm robotics criteria. ${ }^{3}$ Hence, performance of swarm is evaluated based on the proposed modifications. Finally, a new modified algorithm based on combination of the proposed modifications is presented. In addition, the environmental adaptability of the proposed algorithms is investigated.

\section{Honeybee Aggregation Behavior}

Heran ${ }^{19}$ has found that, young honeybees tend to stay in an optimal zone in their comb. The collective behavior of honeybees demonstrated the optimal zone is recognized with temperature in between 34 and $38^{\circ} \mathrm{C}$. Honeybees produce clusters around the optimal temperatures. In this aggregation, honeybee requires a thermal cue.

Regarding to study on aggregation behavior of honeybees, several experiments were performed by Schmickl et al. ${ }^{18}$. In that study, a group of young honeybees ( 1 day old) were placed in a dark arena. In one part of the arena, an IR-lamp was mounted that provided a temperature gradient with non-linear distribution. The arena was divided to four thermal zones, zone A $\left(33-36^{\circ} \mathrm{C}\right)$, zone $\mathrm{B}\left(29-33^{\circ} \mathrm{C}\right)$, zone $\mathrm{C}$ $\left(26-29^{\circ} \mathrm{C}\right)$, and zone D $\left(25-26^{\circ} \mathrm{C}\right)$. Results of the experiments revealed that, density of clustering in nearing with optimal temperature (zone A) was higher than other zones. In addition, the increase of the population size was another effective parameter for achieving the fast aggregation.

In order to study on individual behaviors, 10 bees were selected and their behaviors were captured. Individual behaviors demonstrated that, honeybees are able to distinguish between obstacles and other bees. The second emerged phenomenon from individual 
behavior was the waiting time after each encounter that is happened between two bees. This waiting time was a variable depends on the temperature. Waiting time in zone A was longer in comparison with the other zones. The third interesting behavior of individuals was the inability of a single honeybee to find the optimal zone. Single honeybee had random motion and could not detect the high temperature zone.

Based on the evaluated individual behaviors of honeybees, ${ }^{6}, 18$ those behaviors are fitted in the described criteria of swarm robotics. ${ }^{3}$ Inefficiency of a single bee to detect the optimal zone, simple perception of homogeneous individual agents, and effects of the population size are clearly illustrated in honeybee aggregation. Therefore, bio-inspired BEECLUST algorithm was proposed based on the findings of thermotactic behavior of honeybees (See Refs. 6 and 18 for more details).

\section{Imitation of Honeybee Aggregation}

This section explains description of mobile robot, swarm algorithms, and experimental configurations which are used in this study.

\subsection{Description of AMiR}

Previously, we have introduced an autonomous miniature robot $(\mathrm{AMiR})^{20}$ as an open-hardware swarm robotic platform. It is a small size low-cost platform with feasibility of playing roles in swarm scenarios. The robot is designed with size of 70x70 mm and an AVR micro-controller is deployed as the main processor to control all functions such as communication, trajectory, perception, power management, and user defined tasks. AMiR provides $16 \mathrm{~KB}$ flash memory for programming and $1 \mathrm{~KB}$ internal RAM and 512 byte EEPROM, which are enough memories for swarm applications. Moreover, the micro-controller has eight channels analog to digital converter (ADC) which are used for distance and light intensity measuring. Fig. 1 shows an $\mathrm{AMiR}$ robot that is equipped with a light sensor.

$\mathrm{AMiR}$ is equipped with six IR proximity sensors (in $60^{\circ}$ ) which are used for obstacle detection and also inter-robot communication. ${ }^{21}$ Based on viewing angle of IR components $\left(\sim 60^{\circ}\right)$, the robot covers surrounded area with maximum range of $12 \pm 1 \mathrm{~cm}$. Two micro DC motors with internal gear are utilized for motion of robot with maximum speed of $10 \mathrm{~cm} / \mathrm{s}^{22}$ Power of robot is supplied with a $3.7 \mathrm{~V}$ lithium-polymer battery for autonomy of about 2 hours. A firmware library including basic functions of robot is developed. Opensource gcc compiler (C programming) is selected as the programming language of robot. Latest version of the programming library and compiler are available at: www. swarmrobotic.com.

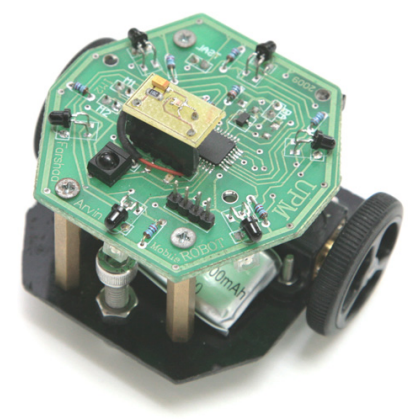

Fig. 1. AMiR mobile robot, open-hardware swarm robotic platform

\subsection{Inspired Algorithm}

The aggregation behavior of honeybee that was described in Section 2 reveals that, agents follow a simple collision-based algorithm ${ }^{6}$ as shown in Fig. 2.

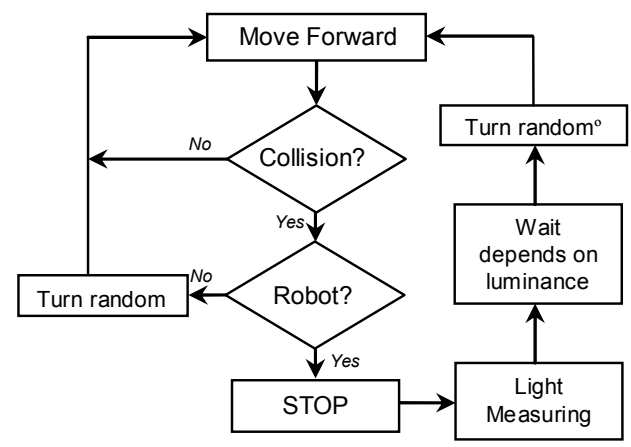

Fig. 2. Algorithm of honeybee aggregation (BEECLUST)

Decisions are made after each collision. This collision does not physically occur between robots chassis. Thus, an inter-robot collision is defined to detect other robot's IR radiations. Robots transmit IR radiation continuously. ${ }^{21}$ Captured values of the IR receivers are checked by the main processor and it produces an interrupt when the IR reaches a defined threshold level. IR reflections and its intensity levels are used to distinguish between wall and other robots. 
When robot detects the IR radiation, it turns off its emitters, and recaptures IR level. If there is IR radiation, it will be another robot. This simple principle helps robots to distinguish between obstacle and other robots. After detecting an obstacle, the robot rotates and executes obstacle avoidance routine. Conversely, if the robot detects another robot, it stops and measures ambient light using light sensor. Robots are equipped with extension lighting sensor for measuring ambient light intensity. This module is placed on top of robot's main board.

A delay function is implemented for calculating the waiting time after each collision. The higher luminance results in a long wait. Waiting time $w(t)$ is a function of luminance, which follows proposed model by Schmickl et al. ${ }^{18}$ :

$$
w(t)=w_{\max } \frac{s(t)^{2}}{s(t)^{2}+\theta}
$$

$w_{\max }$ is the constant value of maximum waiting time for a collision in highest luminance, $S(t)$ is the luminance value of spot which is captured by light sensor, and $\theta$ is the parameter which illustrates the steepness of the waiting curve with respect to luminance. These parameters are estimated based on empirical experiments. For Ref. 18, these values were defined as $w_{\max }=66$ and $\theta=7000$. Estimation of these values is dependent on the robot platform and the experimental configuration.

\subsection{Arena Configuration}

Shape, texture, size, and color of arena are important parameters for analyzing a swarm behavior. It is important that a similar configuration of arena is employed for all experiments. In this study, arena is made out of white body plastic in size of $120 \times 80 \mathrm{~cm}$. Circular covered area by each robot with maximum perception is approximately $800 \mathrm{~cm}^{2}\left(\pi r^{2}\right)$. Six AMiRs are deployed in this study; hence the arena is setup two times larger than area covered by all robots $\left(4800 \mathrm{~cm}^{2}\right)$. Recording robots behavior is important to analyze the social and individual behaviors of robots during each task. For capturing robots' behavior, a digital camera was mounted on top of the arena.

A fluorescent lamp was mounted in one part of arena as the optimal temperature zone. The luminance of arena's light was between 80 and 980 lux as shown in Fig. 3. Similar to the performed experiment in Ref. 6, a static environment with constant value of luminance is employed for all experiments.

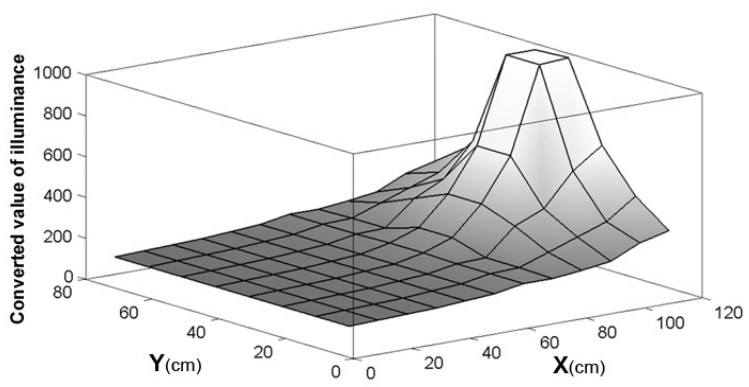

Fig. 3. Spatial distribution of luminance of arena with fluorescent lamp (about 1000 lux)

\section{Experimental Setup}

Experiments include two steps: i) implementation of BEECLUST with AMiR and analysis of the effective parameters in the aggregation performance, and ii) implementation of the proposed modifications and their performance analysis.

\subsection{Honeybee Aggregation}

The first experiment is BEECLUST ${ }^{6}$ that was shown in Fig. 2. In this phase, the effects of several physical parameters relative to inter-robot collision such as number of the participants, arena size, speed of the robots, and sensing threshold are discussed. Waiting time after each collision, $w(t)$, is also another effective parameter on collective behavior of swarm robots.

\subsection{Modified Honeybee Algorithm}

In this phase, two modifications on BEECLUST algorithm are proposed. Dynamic velocity and comparative waiting time are the proposed modifications on original honeybee aggregation. ${ }^{6}$ These modifications should follow the swarm robotics criteria. $^{3}$

\subsubsection{Dynamic Velocity}

The first modification is a function to implement DV that depends on the ambient light. Based on the intensity of ambient light, the arena is divided to three zones. Three levels of velocities are defined for different zones. In area with high luminance (more than 750 lux), 
the robot uses low speed for its motion $(3 \mathrm{~cm} / \mathrm{s})$. This speed determines to stay in the high luminance zone which correspondingly results in more inter-robot collisions in that zone. Robot selects moderate velocity $(6 \mathrm{~cm} / \mathrm{s})$ in the second zone (600-750 lux). In the dark zone (less than 500 lux), robot moves fast to leave that area $(10 \mathrm{~cm} / \mathrm{s})$. For implementing $\mathrm{DV}$, the robot measures ambient light's intensity continuously.

\subsubsection{Comparative Waiting Time}

Second function is CWT that is dependent on intensity of the clustered robots. This behavior is inspired from cockroaches aggregation behavior. ${ }^{16}$ The periodic resting time of cockroaches are changed by cluster's size. This resting time is prolonged based on number of other stationary cockroaches. For CWT, this behavior is combined with BEECLUST for estimating the new waiting time. The robot has 6 IR receivers for recognizing its neighbors. Thus, the robot is able to estimate number of the surrounded robots $(\varphi)$ after each collision. In this case, waiting time is defined as a function of luminance and number of neighbors:

$$
w_{d}(t)=\varphi \cdot w(t)
$$

where $w_{d}(t)$ is the estimated waiting time for CWT which updates previous waiting time function (Eq. 1) depends on the neighboring robots. Due to the IR components topology in main board, $\varphi$ is between 1 and 4. In order to prevent clustering in the low luminance zone, CWT is applied only in the optimal zone with the higher luminance than 750 lux. In area with luminance between 500 and 750 lux, $\varphi$ is not estimated and the constant value of 1 is assigned. Fig. 4 illustrates the waiting time for the proposed CWT as a function of luminance. This function helps the robots to stay for a long period in the high luminance area (optimal zone).

\subsection{Number of Inter-Robot Collision}

The robots in aggregation scenario should detect other robots and stay with them to allow another robot to recognize them. The number of inter-robot collisions is an important parameter for the prediction in collisionbased processes. Kernbach et al. ${ }^{6}$ defined a relationship using Eq. 3 to estimate the number of inter-robot collisions following the aggregation behavior. This relationship uses diffusion behavior of molecularkinematic theory in ideal gas.

$$
n_{n}=\frac{2 \sqrt{2} R_{s} v t N}{S_{s w}-N S_{r}}
$$

$n_{s}$ is the number of inter-robot collisions for an individual robot, $R_{s}$ is the robot's sensing radius, $v$ is the robot's speed, $t$ is the aggregation time, $N$ is the number of robots, $S_{s w}$ is the size of the experiments' arena, and $S_{r}$ is the robot's size.

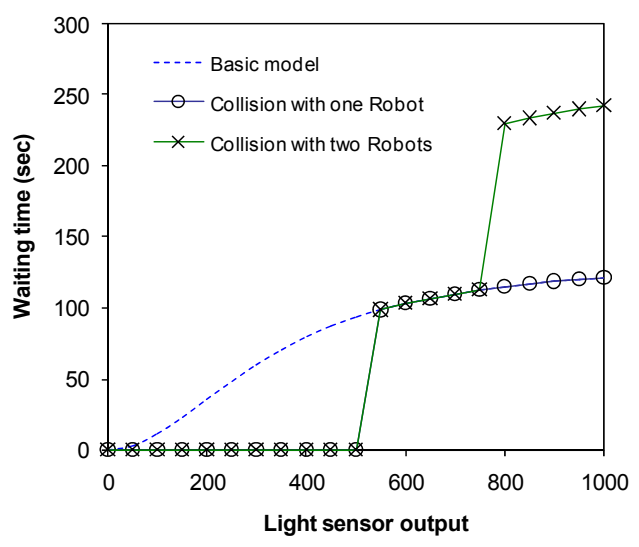

Fig. 4. Estimated waiting time using CWT function depends on luminance and number of neighbors

\subsection{Sampling and Statistical Analysis}

In order to data validation, each experiment was repeated 20 times and an experiment with duration of longer than 15 minutes was assumed as a null experiment. The successful aggregation is defined as an experiment that all robots are gathered in the light zone with the high luminance (>750 lux).

For statistical analysis, one-way ANOVA was used to test the significance of the parameters in aggregation behavior. The $P$-value shows the individual significance probability of each independent variable. The term with $P<0.005$ is considered to be statistically significant.

\section{Results and Discussion}

\subsection{Honeybee Aggregation}

This experiment was performed with individual groups of $2,3,4,5$, and 6 robots in two different arena sizes: i) small 60x80 cm and ii) large 120x80 cm. Time required for aggregation at the optimal zone with respect to the robot number is shown Fig. 5. As shown in the obtained 
results, the number of participants significantly increases the efficiency of the swarm in both area sizes ( $F=32.45, P<0.005)$. Based on the defined time-out for each experiment, the individual groups of 2 and 3 robots could not aggregate in the large arena. This phenomenon proves the inefficiency of the low populations to solve a joint problem. ${ }^{3}$ In small size arena, two robots also could aggregate though it was not a stable aggregation. The number of collisions for each individual robot was not changed significantly $(P>$ $0.005)$ depending on the number of the robots. Similar behavior was reported in Ref. 6 .

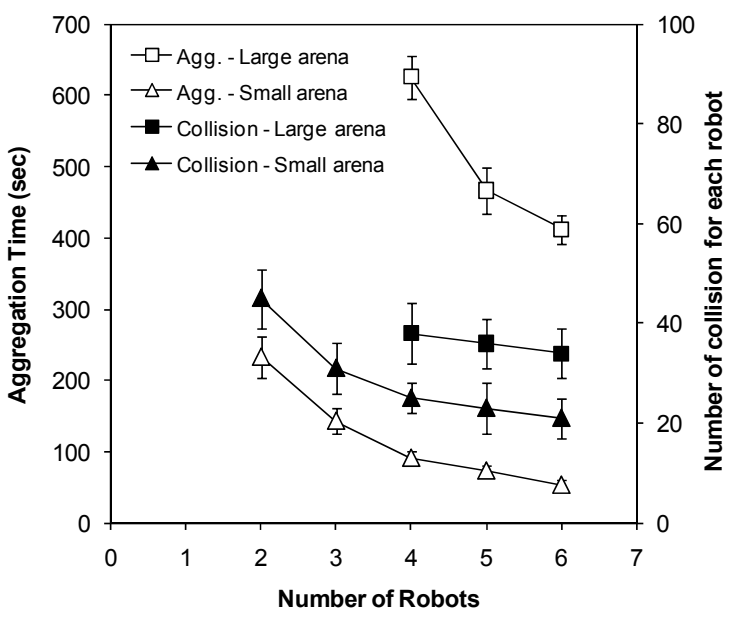

Fig. 5. Aggregation time and number of collision for each robot versus the population size in two arena sizes.

Another important sensory parameter was the threshold level for the robot's environmental perception. Fig. 6 illustrates the effects of sensing threshold of collision detection in different aggregation experiments with 6 robots. The results showed that, the suitable threshold range is between 300 and 500. In thresholds higher than 500, robots must be close together for collision to occur. In values less than 300 , robots detect the collision in a far distance of the clustered robots which resulted in stopping in the dark zone. Fig. 7 reveals the aggregation of six robots using two threshold levels of 200 and 600.

Another important parameter that contributes to the efficiency of the swarm was the waiting time after each inter-robot collision. The aggregation time is long when the waiting time is defined short. During the long waiting time, clustered robots are stationary for the long period and so chance for other robots to contact them is increased. Prolongation of the waiting time will not help in completing the task much faster due to limitation of the robot's velocity and the sensory system.

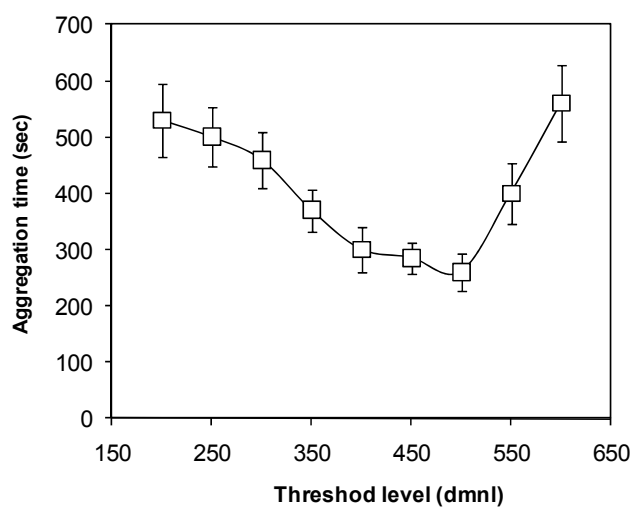

Fig. 6. Aggregation time relative to sensitivity level of sensors in detecting other robots (performed with 6 robots).

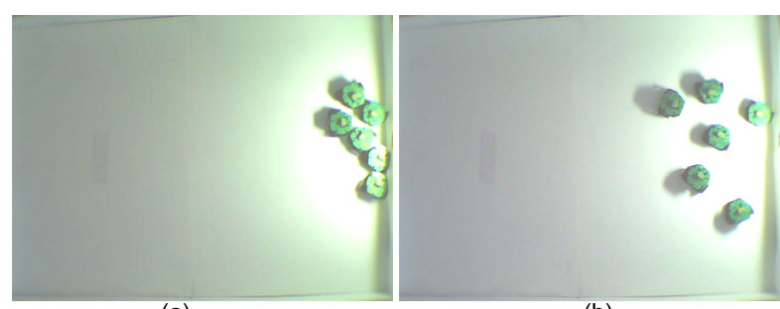

(a)

(b)

Fig. 7. (a) Aggregation with threshold level of 600 and (b) aggregation with threshold level of 200

The goal is to find an appropriate $w_{\max }$ and to estimate a suitable $\theta$ for waiting time model (Eq. 1). In this regard, six AMiRs were participated in the large size arena with the luminance distribution as shown in Fig. 3. Fig. 8 shows the aggregation time with respect to different values of $w_{\max }$. The suitable $w_{\max }$ which is happened in the highest luminance (in 980 lux) was about $135 \mathrm{sec}$. This value is two times longer than model values in Ref. 18 due to robot's speed and arena configurations. After several empirical experiments, the model of waiting time using the new values is shown in the following formula:

$$
w(t)=135 \frac{s(t)^{2}}{s(t)^{2}+112500} .
$$

Fig. 9 shows the effects of different velocities of the robot in aggregation behavior. Maximum speed of the robot was about $10 \mathrm{~cm} / \mathrm{sec}$. As expected, velocity of the robot directly influenced the aggregation time. This experiment was also performed with the long waiting 
time (about 2 times). Therefore, the effects of velocity in the long waiting time were smoother than the previous experiment. In slow robots, when a robot was stationary for a long period, other robots found the chance to reach the immobile robot.

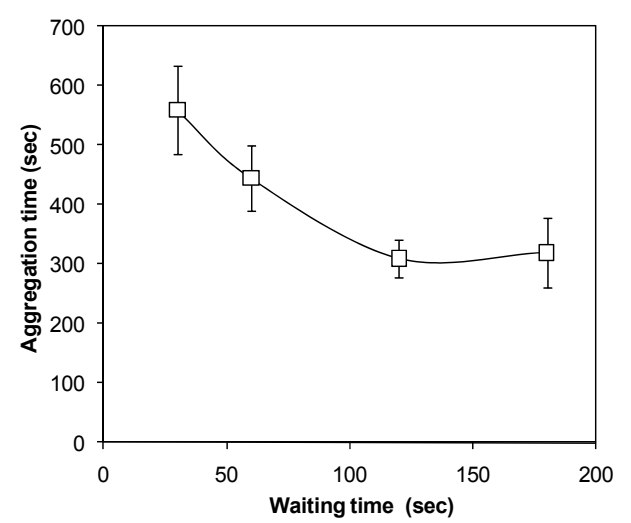

Fig. 8. Aggregation time for six AMiRs as a function of waiting time

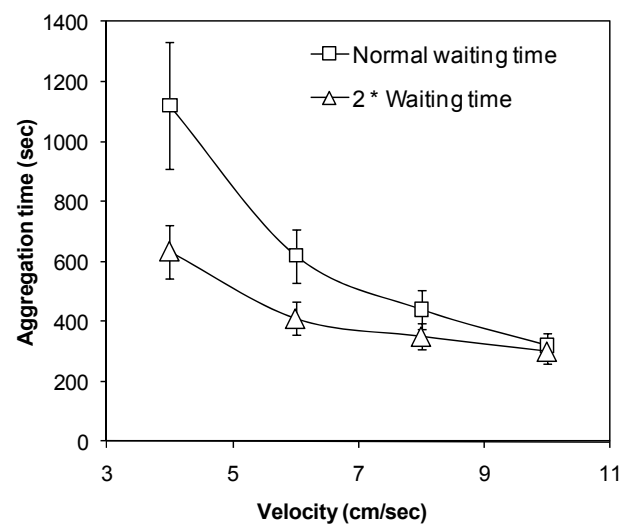

Fig. 9. Aggregation time as a function of velocity with two waiting times

Fig. 10 shows captured images of an aggregation behavior in time intervals of $75 \mathrm{sec}$. This experiment was performed using the specific parameters of the algorithm $\left(w_{\max }=135, \theta=112500\right.$, sensors' threshold level of 440 , and maximum velocity of $10 \mathrm{~cm} / \mathrm{sec}$ ). The aggregation was achieved around $400 \mathrm{sec}$ in the large size arena.

The robots could solve the problem using simple perception without requiring explicit communication. The collective behavior of the robots could show that, BEECLUST is an acceptable algorithm to be used as a swarm behavior. Schmickle et al. ${ }^{18}$ have demonstrated the practicality of BEECLUST with Jasmin microrobot. Swarm behaviors must be independent from the platform. Hence, the first phase of our experiments demonstrated the BEECLUST is not dependent to a given platform and it appropriately follows the swarm robotics criteria. ${ }^{3}$ In the second phase, we would like to investigate the performance of the swarm with simple algorithmic modifications on the original BEECLUST.

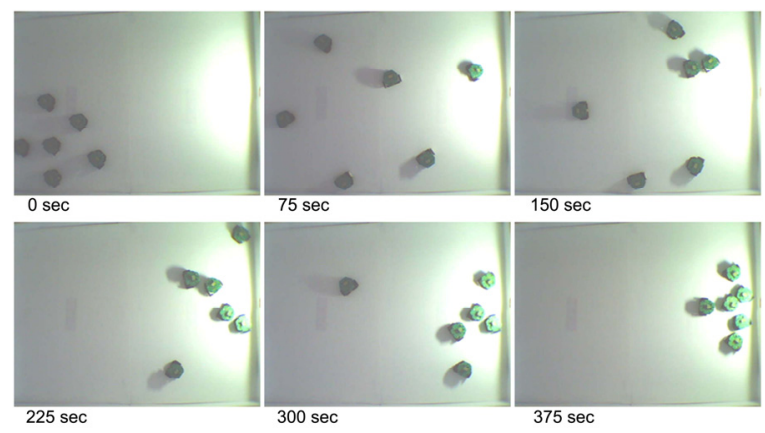

Fig. 10. A group of six AMiRs could find the optimal zone.

\subsection{Modified Honeybee Algorithms}

In this section, results of the aggregation scenarios with proposed modifications are presented. The obtained results are statistically analyzed and effects of each algorithm are discussed.

\subsubsection{Dynamic Velocity}

This function allows robot to leave the dark zone rapidly. On the other hand, motion with minimum speed in the high luminance zone increases the number of robots in that zone, thus most collisions are occurred in the optimal zone. Experiments were performed with various numbers of robots to check the swarm scalability. Fig. 11 illustrates the aggregation time for modified and original algorithms. Although the number of collisions was not changed significantly, the aggregation times were reduced significantly ( $F=46.98$, $P<0.005)$. The most imperative difference among the original and modified algorithms is the occurring collisions in the high luminance zone using DV function. Therefore, the scenario could be accomplished in shorter time than original BEECLUST. This function could reduce swarming time for a group of 6 robots from $400 \mathrm{sec}$ to around $300 \mathrm{sec}$. In addition, group of 3 robots could complete the scenario in around $600 \mathrm{sec}$. 


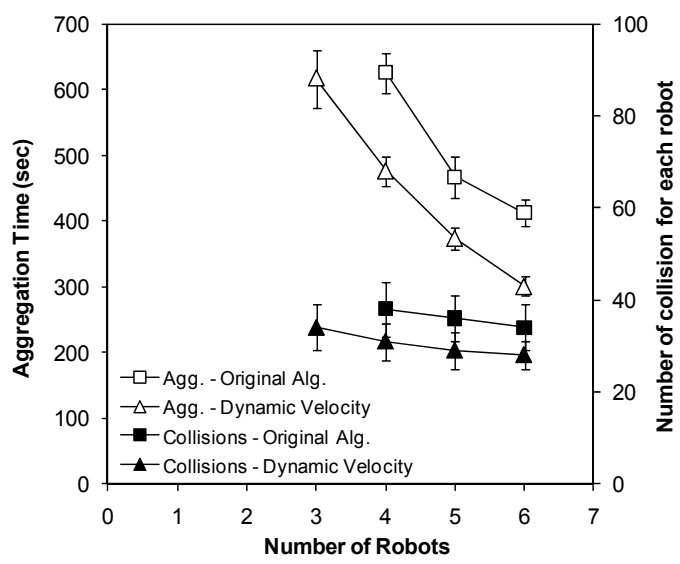

Fig. 11. Aggregation time and number of collision for BEECLUST and dynamic velocity algorithms

\subsubsection{Comparative Waiting Time}

The second experiment was the study on the effects of CWT. This function changes the waiting period of inter-robot encounters based on number of the neighboring robots. The luminance of the zone is another effective parameter for estimating the new waiting time. Fig. 12 reveals the effects of CWT on aggregation time of swarm robots. Statistical analysis of the obtained results showed that, the aggregation time was significantly reduced $(F=71.40, P<0.005)$. Due to the long waiting time of the robots in the optimal zone, number of inter-robot collisions was decreased and collisions usually resulted in stationary in the optimal zone. Similar effects of the resting time were reported in other aggregation researches. ${ }^{16,18}$ A group of six robots could aggregate at the light area in $200 \mathrm{sec}$.

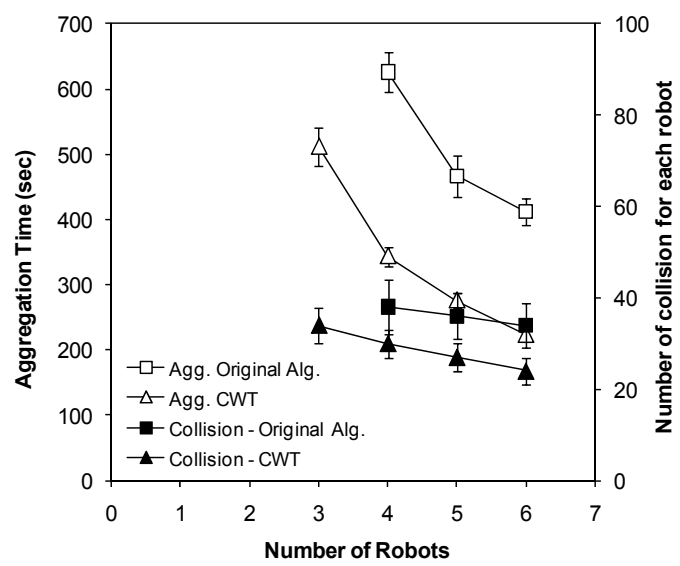

Fig. 12. Aggregation time and collision of each robot for BEECLUST and comparative waiting time

\subsection{New Aggregation Algorithm}

A new aggregation algorithm with combination of the described modifications was proposed. Fig. 13 illustrates the aggregation time for the original and proposed modifications. Statistical analysis of the results showed that the combined algorithm was significantly reduced the aggregation time $(F=91.16, P$ $<0.005)$. Based on the $F$-value, the combined algorithm of DV and CWT was high effective than others in swarm performance.

Therefore, a new aggregation algorithm with combination of the performed modifications is determined as shown in Fig. 14.

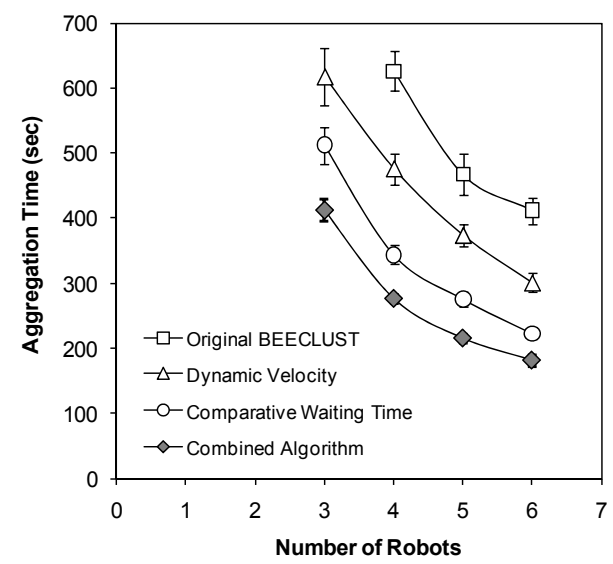

Fig. 13. Aggregation time for original BEECLUST and modified algorithms

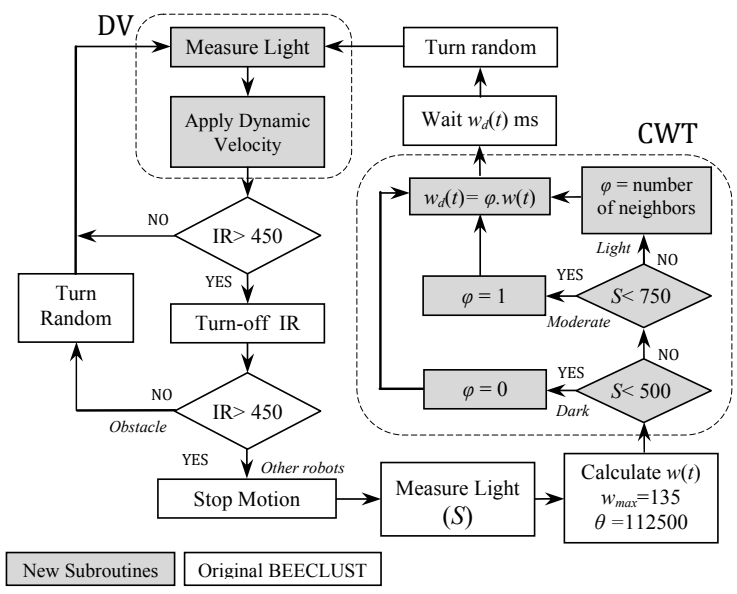

Fig. 14. Proposed aggregation algorithm based on combination between performed modifications 


\subsection{Number of Inter-Robot Collisions}

As for the number of inter-robot collisions represented by Eq. 3, Fig. 15 shows an approximation model that depicts collision number as a function of population size for different algorithms. Aggregations using the proposed modifications were accomplished with small $n_{s}$ than original BEECLUST. These differences were occurred due to the experimental configurations. The important point is the convergent behavior for $n_{s}$. The convergent behavior of $n_{s}$ is shown in all algorithms with different mean values. As reported in Ref. 6, the fluctuation of $n_{s}$ in each experiment was explained by a systematic inaccuracy. In the cases of CWT and combined algorithms, $n_{s}$ were fluctuated more than others with low-population sizes. Based on the expectation, this can be compensated with large scale experiments. The most important point is the constant value of $n_{s}$ for all performed algorithms. Similar to the reported results in Ref. 6, ns was independent of parameters such as population, arena sizes and platform. Hence, the modified aggregation scenarios performed in this study are the scalable swarm algorithms.

As it was expected, the total number of collisions that were occurred during each experiment increased with increasing populations $\left(N_{\mathrm{t}}=N \times n_{\mathrm{s}}\right)$.

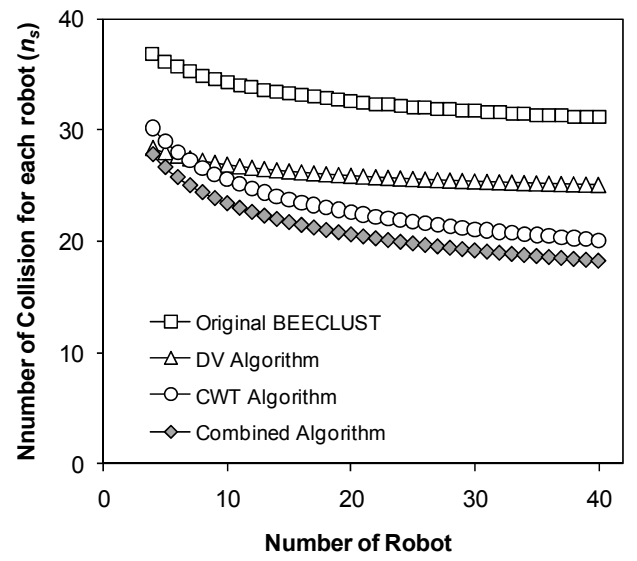

Fig. 15. Estimation of collision number for a single robot as a function population size

\subsection{Environmental Adaptability}

Finally, the adaptability of the proposed algorithms to the environmental fluctuations was investigated. In this regard, after an accomplished aggregation for 6 robots the position of the fluorescent lamp was changed to another side of arena. Fig. 16 shows number of the aggregated robots during re-aggregation scenarios. The experiments were repeated 20 times for each algorithm and mean values of the population sizes were calculated. Clearly, after changing the lamp position, the robots wait to finish their waiting times. These waiting times for CWT and combined algorithms are long period due to their definitions. However, these algorithms could aggregate around new light position faster than others. Therefore, the proposed algorithms are able to adapt to the environmental fluctuation.

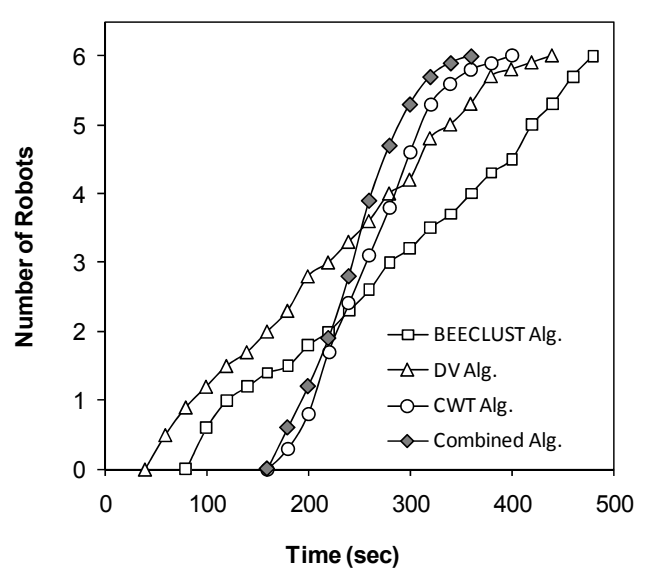

Fig. 16. Average number of the aggregated robots after changing lamp position for different algorithms as a function of time

Proposed modifications in this study increased the sensing ability of the individual robots slightly. Hence, fast aggregation using these modifications was expected. This phenomenon was demonstrated in the reduced number of collisions for each individual robot. It showed that, the most collisions of the robots were occurred in optimal zone. The main objective of this study was to demonstrate that, a small modification on behavior of individual robots results in big changes in collective behavior of swarm. This change helps us to apply various inspired behaviors of social insects or animals for solving a particular problem using simple modifications on original algorithms.

\section{Conclusion}

In this paper, an imitation of honeybee aggregation with miniature size mobile robots was illustrated. The first phase of experiments was the implementation of BEECLUST using AMiR. The studied parameters were 
arena size, number of the robots, sensitivity and velocity of the robots, and waiting time after inter-robot collision. Based on thermotactic behavior of honeybees, two algorithmic modifications in decision making stages were proposed. Dynamic velocity and comparative waiting time were these modifications. Results showed the proposed modifications were significantly reduced the aggregation time. A new aggregation algorithm using the combination of the performed modifications was presented. In addition, the adaptability of the algorithms to the environmental fluctuation was tested with changing the position of optimal zone. The results of this study concluded that, it is possible to define a new swarm behavior with modification and combination of inspired algorithms without missing the criteria.

\section{References}

1. E. Şahin and A. Winfield, Special issue on swarm robotics. Swarm Intelligence, 2(2) (2008) 69-72.

2. E. Şahin, Swarm robotics: From sources of inspiration to domains of application. Lecture Notes Computer Science, ed. E. Şahin and W.M. Spears. Vol. 3342. 2005.

3. M. Dorigo and E. Şahin, Swarm robotics - special issue editorial. Autonomous Robots, 17(2) (2004) 111-113.

4. C. Melhuish, A. Sendova-Franks, S. Scholes, I. Horsfield, and F. Welsby, Ant-inspired sorting by robots: the importance of initial clustering. Journal of The Royal Society Interface, 3(37) (2006) 235-242.

5. S. Alavandar, T. Jain, and M. J. Nigam, Bacterial Foraging Optimized Hybrid Fuzzy Precompensated PD Control of Two Link Rigid-Flexible Manipulator International Journal of Computational Intelligence Systems, 2(1) (2009) 51 - 59.

6. S. Kernbach, R. Thenius, O. Kernbach, and T. Schmickl, Re-embodiment of Honeybee Aggregation Behavior in an Artificial Micro-Robotic System. Adaptive Behavior, 17(3) (2009) 237.

7. Y. Shen, G. Wang, and C. Tao, Particle Swarm Optimization with Novel Processing Strategy and its Application. International Journal of Computational Intelligence Systems, 4(1) (2011) 100 - 111.

8. X. You, S. Liu, and Y. Wang, Quantum Dynamic Mechanism-based Parallel Ant Colony Optimization Algorithm. International Journal of Computational Intelligence Systems, 3(1) (2010) 101 - 113.

9. W. Wang, Q. Sun, X. Zhao, and F. Yang, An improved Particle Swarm Optimization Algorithm for QoS-aware Web Service Selection in Service Oriented Communication. International Journal of Computational Intelligence Systems, 3(1) (2010) 18 - 30.
10. O. Soysal and E. Şahin. Probabilistic aggregation strategies in swarm robotic systems. in Proc. of the IEEE Swarm Intelligence Symposium (2005) 325-332.

11. H. Çelikkanat and E. Şahin, Steering self-organized robot flocks through externally guided individuals. Neural Computing \& Applications, 19(6) (2010) 849865.

12. S. Garnier, C. Jost, J. Gautrais, M. Asadpour, G. Caprari, R. Jeanson, A. Grimal, and G. Theraulaz, The embodiment of cockroach aggregation behavior in a group of micro-robots. Artificial Life, 14(4) (2008) 387408.

13. C. Kube and H. Zhang, Collective robotics: From social insects to robots. Adaptive Behavior, 2(2) (1993) 189.

14. C. Melhuish, O. Holland, and S. Hoddell, Convoying: using chorusing to form travelling groups of minimal agents. Robotics and Autonomous Systems, 28(2) (1999) 207-216.

15. T. Schmick1, C. Moslinger, and K. Crailsheim, Collective perception in a robot swarm. Lecture notes in computer science, 4433 (2007) 144.

16. S. Garnier, J. Gautrais, M. Asadpour, C. Jost, and G. Theraulaz, Self-Organized Aggregation Triggers Collective Decision Making in a Group of CockroachLike Robots. Adaptive Behavior, 17(2) (2009) 109.

17. R. Jeanson, C. Rivault, J. Deneubourg, S. Blanco, R. Fournier, C. Jost, and G. Theraulaz, Self-organized aggregation in cockroaches. Animal Behaviour, 69(1) (2005) 169-180.

18. T. Schmickl, R. Thenius, C. Moeslinger, G. Radspieler, S. Kernbach, M. Szymanski, and K. Crailsheim, Get in touch: cooperative decision making based on robot-torobot collisions. Autonomous Agents and Multi-Agent Systems, 18(1) (2009) 133-155.

19. H. Heran, Untersuchungen uber den Temperatursinn der Honigbiene (Apis mellifica) unter besonderer Berucksichtigung der Wahrnehmung strahlender Warme. Journal of Comparative Physiology A: Neuroethology, Sensory, Neural, and Behavioral Physiology, 34(2) (1952) 179-206.

20. F. Arvin, K. Samsudin, and A. Ramli, Development of a Miniature Robot for Swarm Robotic Application. International Journal of Computer and Electrical Engineering, 1 (2009) 436-442.

21. F. Arvin, K. Samsudin, and A. R. Ramli, Development of IR-Based Short-Range Communication Techniques for Swarm Robot Applications. Advances in Electrical and Computer Engineering, 10(4) (2010) 61-68.

22. F. Arvin, K. Samsudin, and M. A. Nasseri. Design of a differential-drive wheeled robot controller with pulsewidth modulation. in Innovative Technologies in Intelligent Systems and Industrial Applications, CITISIA (2009) 143-147. 